\title{
Analysis of Spectrum Selection Methods based on Platform-Qualnet in Cognitive Radio Networks
}

\author{
https://doi.org/10.3991/ijoe.v15i03.9517 \\ Hui Sun $\left.{ }^{\varpi}\right)$, Han Han \\ Civil Aviation University of China, Tianjin Shi, China, \\ shhappy1@ hotmail.com
}

\begin{abstract}
In this paper, we compare two different spectrum assignment methods in cognitive radio network. The scheme includes channel selection with control channel and non-control channel. A control channel method termed as full information channel selection is discussed and compared with receiverdirected channel selection based on the simulation on Qualnet platform. We also consider the impacts from malicious users (MU) attack in two conditions. The results prove that the receiver-directed channel selection can match to the throughput of control channel selection and reduce the attack probability.
\end{abstract}

Keywords - Cognitive radio network, Control channel, Channel selection

\section{Introduction}

To solve the radio spectrum resource scarcity, the dynamic spectrum access technology is proposed [1][2]. In DSA, secondary user (SU) detects the spectrum channels and accesses the available spectrums for communication randomly. As a specific example with this technology, cognitive radio network needs each user to detect available spectrum and selects the optimal one as its operating frequency independently. Cognitive radio is a kind of essentially software defined radios with builtin artificial intelligence, capable of sensing and reacting to a network's environment. This process of assigning a channel to each radio interface as its operating frequency is termed spectrum assignment [3]. Spectrum sensing, spectrum decision, spectrum sharing, and spectrum mobility are important components in spectrum management. The basic structure is shown in Fig. 1.

In a cognitive radio network, two kinds of users are considered. The primary user (PU) has the license to use the spectrum and accesses the spectrum without limitation. The secondary user (SU) could access the spectrum when PU is not exit. And secondary user has to evacuate from the current spectrum to avoid impact PU access if primary user accesses the same spectrum [2]. Base on this characteristic, the spectrum sensing is very important for the SU to use the available spectrum. Generally, spectrum sensing includes distributed and centralized modes. In the first case, SU gets the spectrum information from their neighbors. In the second case, SU receives the spectrum information from the control channel, which selects the knowledge of spectrum from all SUs in the network. 
Due to the unique feature of the cognitive radio network, it is also vulnerable to attacks from malicious user, especially if the cognitive radio network has the control channel. In this case, if the control channel is attacked, the SUs in this network could not get the useful information about spectrum. They could not access the empty spectrum or have to leave the occupied spectrum with fake information. Also, the cognitive radio network cannot avoid some common attacks, which happen on the traditional wireless networks [4].

Simulation software is a very important tool to learn wireless networks. Compare to the physical test, the software simulation method is more effective and flexible. Qualnet is a well-known network simulation software developed by Scalable Network Technologies and used by many companies such as Microsoft, Lockheed Martin, the United States Air Force, etc. Qualnet is a performance prediction software capable of representing physical and wireless networks as software virtual networks in real time speeds [5] [6]. Qualnet is designed based on realistic performance measures. Also, Qualnet offers many tools to the learners make simulating and testing process easy and accurate.

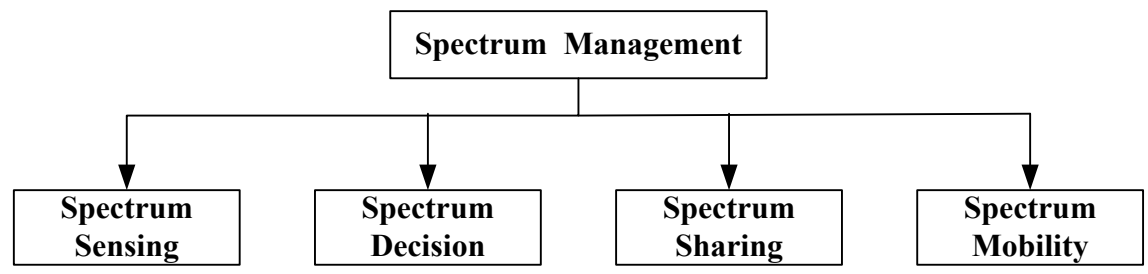

Fig. 1. The architecture of spectrum management

In the following sections, the basic models of cognitive radio network users are introduced in section 2. Afterward, section 3 explains two different methods which are implemented in channel assignment. Thereafter, some simulation results are compared in section 4. In the last section, a conclusion is given.

\section{System Model}

\subsection{Primary user}

Primary user has a license to occupy channel and forces the secondary users to leave the occupied channel if it accesses to the channel. We assume that the arrival of primary user activities in each channel is simulated as a Poisson process with arrival rate $\lambda$ and the duration of occupying channel by a primary user is exponentially distributed with mean $1 / \mu$. 


\subsection{Secondary user}

Secondary user detects spectrums by using energy detect method [7] [8] and selects the optimal spectrum to communication. In this paper, we assume the secondary user can select available spectrum based on its neighbors or from the control channel. If the spectrum is empty, the secondary user communicates on this channel. And, the secondary user should withdraw from communication when primary user or malicious user accesses the channel.

\subsection{Spectrum / Channel}

When a primary user or malicious user access a spectrum, the state of the spectrum is busy for the secondary user. Otherwise, the state of the spectrum is idle or available for secondary user access.

If a PU accesses a spectrum, it covers a circular area in the network, with a radius uniformly distributed between 0 and the network diameter. All users within this circle detects this channel as inaccessible.

\section{Channel Selection Methods and Simulation}

\subsection{Full information channel selection (FIC)}

In this spectrum selection scheme, all users have the knowledge of the neighbors who use the control channel. If a secondary user changes its operating spectrum, it should broadcast to all of its neighbors to notify the new spectrum. When a neighbor receives the information, it records it in its list. If a secondary user wants to send packets, it checks the list to find the spectrum which neighbor is current on, then switches to that channel and communicates with other user.

\subsection{Receiver-Directed channel selection (RD)}

In the RD approach, at each time slot, secondary user is on a specific channel to receive packets. If a PU arrives on user's specific channel, that user can no longer use this channel for communications and should determine a new one. When secondary user desires to transmit packets to another user, it switches to the specific spectrum of that destination user occupies. It is possible that a user switches to the channel of the original user, but at this time, the original user is not on its channel. To avoid this issue, we suppose that secondary user either stays on its specific spectrum or switches to other user's specific spectrum in a given time slot [9]. To implement this, we should evaluate the state of the secondary user in a given time slot. If the state is ' 1 ' then user stays on its specific channel to receive packets from other users. Otherwise, the secondary user switches to the spectrum of another user to communication. 


\subsection{Simulation with Qualnet}

Qualnet provides us with the ability to implement these channel assignments and collect valuable data on the network performance. In this scenario, each user has a single radio, known as an interface, as a default. And the user is capable of adding additional interfaces to the user as desired. However, each interface is only capable of half-duplex communications. The problem with this system occurs if a user attempts to send messages. To simulate and make sure it yields realistic results, it is supposed to have packets which are produced on the application layer. Then packets are passed down through the OSI model. To accomplish this purpose, an application file is called within the .config file. And it specifies the type of application, source, and destination of the packets to start or stop producing these packets, and so on. At the application layer, a generic traffic packet generator is chosen to produce these packets. Since the channel assignments schemes reside in the network layer, these packets are received at the router function which either passes them along to a suitable neighbor or stores the packets in a queue for processing until a suitable neighbor is found.

During the simulation process of cognitive radio networks, the PU activity simulation is an important component. Assume that a PU occupies on a channel, it covers a circular area with a radius range from 0 to the network diameter. The example of this topology is shown in Fig. 2. And in this topology, all users within this circle detects this channel as inaccessible.

In this paper, the SU is used to generate PU activities in the simulation environment as follows.

- Specifically, a timer is set in the first SU node. Whenever this timer expires, a PU activity is generated by the first SU node, with a scheduled arrival time and departure time. These parameters are generated by a random generator in Qualnet.

- The arrival activity of PU in each channel is simulated as a Poisson process with arrival rate $\lambda$ (per second). The duration of a PU activity is exponentially distributed with mean $1 / \mu$ (seconds).

- Furthermore, two random generators are used to create the axis values of the simulation field and the transmission range for PU activity, respectively.

Based on these three sets of values mentioned above, a PU activity could be placed on the simulation field with its arrival or departure time on random location in a coverage area.

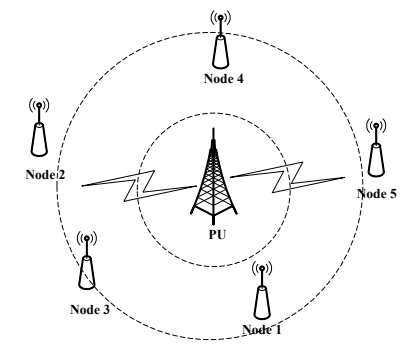

Fig. 2. Cognitive radio network example 


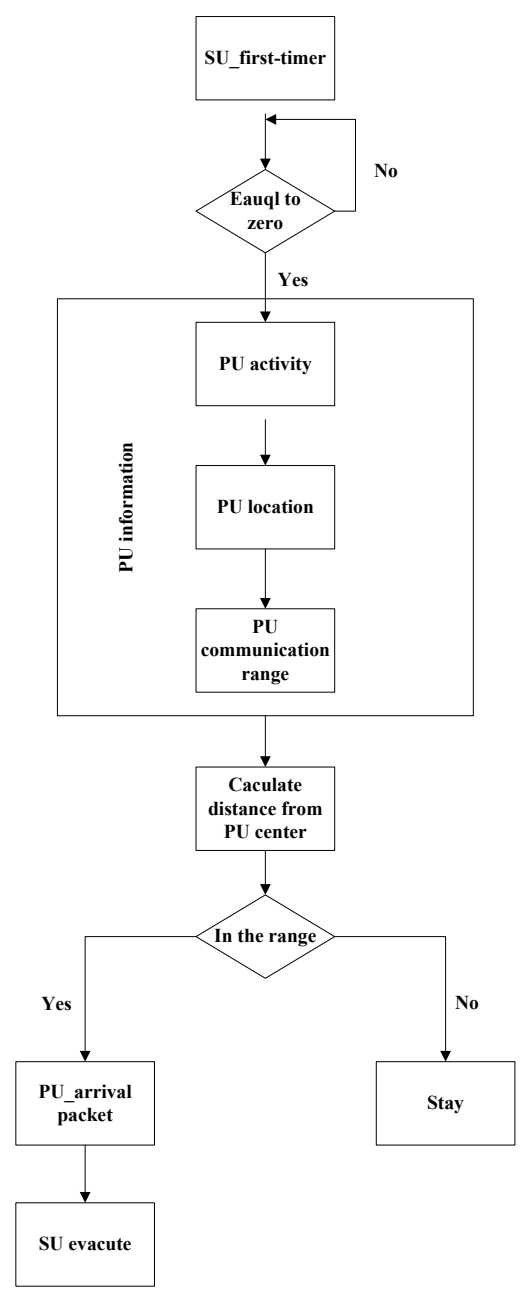

Fig. 3. Flowchart of the protocol

At last, the first SU calculates the distance of each user to the PU activity center. If the user is within the coverage area of the PU activity, the first user sends a "PUarrival" packet including the information of a channel used by PU to SUs over the second radio interface. After the destined SU receives this packet, it takes the header off and reveals the content of the packet. Because the content of the packet is about the current channel which is used by the PU, the SU would stop its data communications and leave this channel immediately until the corresponding PU departs the simulation field. In the meantime, if the first user would send a "PU-departure" packet to the same user, the receiving user enables the channel to communications again. The basic idea is illustrated in Fig. 3. A single-user channel has only one user on it and thus the user cannot communicate with other users. For secondary user pair, When they finds a utilized channel, they start sending any data packets [10] [11] [12]. 


\section{Simulation Results}

In this paper, IEEE $802.11 \mathrm{~b}$ is used as the MAC protocol. Each channel assignment scheme uses a header file that is associated with it. This header file holds the data structures needed, function prototypes, and various parameters that dictate how the protocol behaves during a simulation.

\subsection{Users in a grid topology}

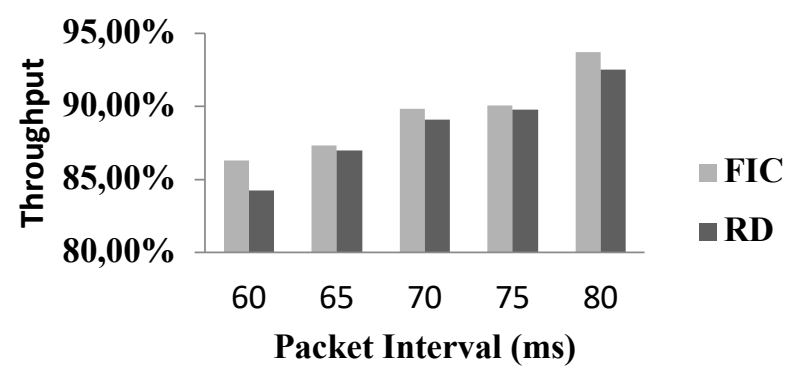

Fig. 4. Comparison of throughput between RD and FIC in a grid topology

In Fig. 4, FIC has the highest network throughput. The reason is, in FIC, all users already know their neighbors' communication spectrum. Comparing with the RD scheme, a slight drop in performance compared to that of FIC. The difference between RD and FIC happens because secondary users must wait for the proper combination of states between users in order to transmit data packets in the RD method. And, the lower the interval the more packets being created and potentially flooding the network layer causing interference hence, the performance drop.

\subsection{Nodes in random topology}

Compare with the grid topology, in random topology, the user has a large number of neighbors at any given time. But, packet loss could happen if there are multiple neighbors on the same channel at a given time. In Fig. 5, we can see the performance is close between RD and FIC as illustrated in Fig 5. 


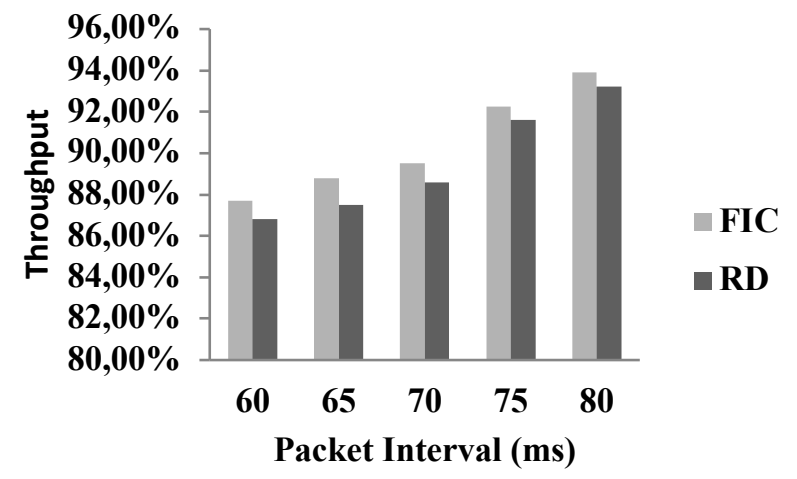

Fig. 5. Comparison of throughput between RD and FIC in random topology

\subsection{Channel Switch Success Probability}

FIC is an approach with the control channel. If the control channel is attacked by the malicious user which emulates the primary user's signal characteristics, the secondary user receives the fake information and makes wrong decision. The RD method does not need a control channel, but the secondary user should switch the channel to make communication. So, the success probability of channel switch must be considered. In this paper, if a secondary user connects with the original pair after switching channel, the channel switch is regarded as a successful switch.

A channel switch success probability means the probability that secondary user makes a successful connection to other users with the switching the channel. The probability can be defined as below.

$$
P_{s}=\frac{C_{s}}{C_{t}}
$$

Where, $P_{s}$ is the channel switch success probability, $C_{s}$ and $C_{t}$ are total channels number and switching channels number, respectively. 


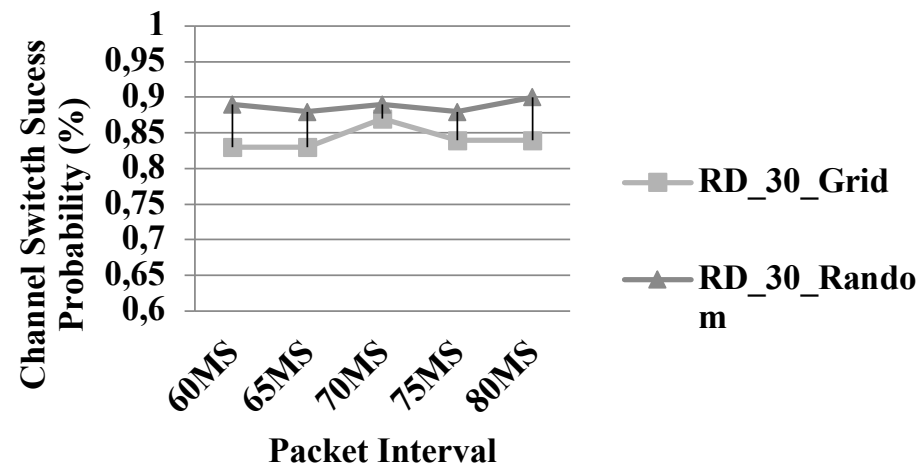

Fig. 6. RD channel switch success probability

Fig. 6 illustrates that the channel switch success probability is around 0.9 by using $\mathrm{RD}$ channel selection scheme. And the performance in random topology is better than that in a grid topology. The simulation results prove that the RD method has a high success channel switch probability to implement the data transmission.

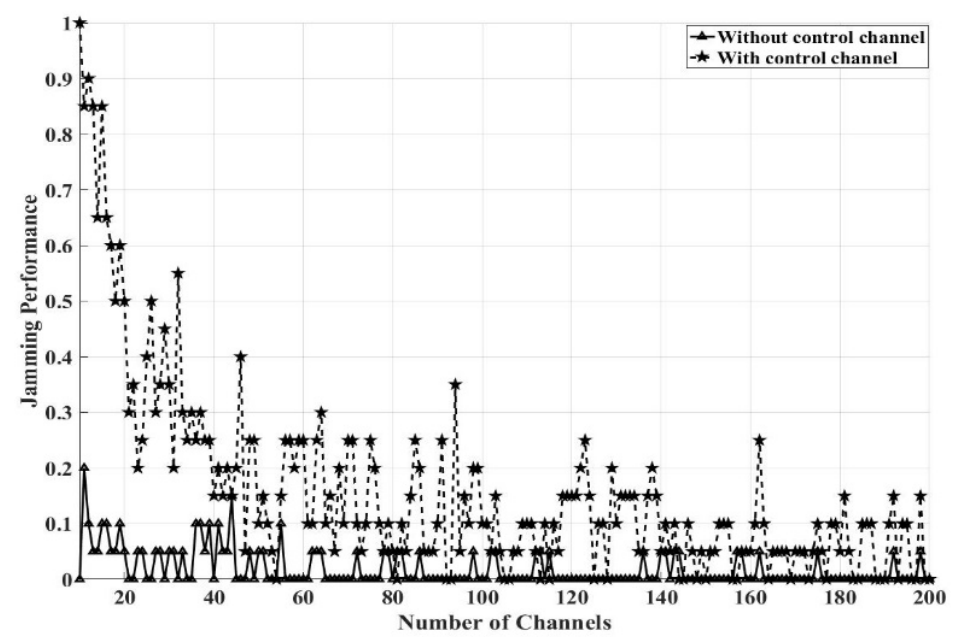

Fig. 7. Comparison of jamming performance in two cases

If malicious user attacks the cognitive radio network, communication between secondary user pair is jammed. In Fig. 7, the comparison is shown based on different situations. With the increase of channel numbers, the jamming probability decreases since the malicious user attack the specific channel difficulty. But, the jamming performance without a control channel is better than that of the situation with the control channel. 


\section{Conclusion}

Control channel helps secondary users to share the spectrum state information and shows high throughput performance in the cognitive radio network. But, as a fusion center, the control channel cannot transmit data among secondary users. On the other hand, since the control channel relates to the whole system, if the control channel is impacted, the performance of the network becomes worse. RD method is considered as a approach to solve this issue in this paper, and Qualnet is used to construct virtual networks and simulate packets transmission. The simulation results validate that the design used by the RD channel assignment mechanism can reach network throughputs close to that of FIC with high success channel switch probability. And can reduce the risk of attack from malicious users.

In the future work, the performance of the dynamic spectrum access network under attack by malicious users is considered in RD channel selection scheme.

\section{References}

[1] I. F. Akyildiz, W.-Y. Lee, M. C. Vuran, and S. Mohanty(2006). NeXt generation/dynamic spectrum access/cognitive radio wireless networks: A survey. Comput. Netw., Int. J. Comput. Telecommun. Netw., vol. 50, no. 13, pp. 2127-2159, Sep. https://doi.org/1 0.1016/i.comnet.2006.05.001

[2] B. Wang and K. J. R. Liu (2011). Advances in Cognitive Radio Networks: A Survey. IEEE J. Sel. Topics Signal Process. vol. 5, no. 1, pp. 5-23. https://doi.org/10.1 109/JSTSP.2010.2093210

[3] R. Chen and J. M. Park (2006). Ensuring trustworthy spectrum sensing in cognitive radio networks. Proceedings, IEEE Workshop on Networking Technol. For Software Defined Radio Networks (SDR) 2006, pp. 110-119.

[4] John T. MacDonald, D. A. (2007). Spectrum Occupancy Estimation in Wirless Channels with Asymmetric Transmitter Powers. 2nd International Conference on Cognitive Radio Oriented Wireless Networks and Communications.

[5] Technologies, Scalable Network. (n.d.). Scalable Network Technologies. Retrieved from Qualnet developer: http://www.qualnet.com/products/developer.php

[6] QualNet Documentation, "QualNet 5.0 Model Library: Advanced Wireless": http://www.scalablenetworks.com/products/Qualnet/download.php\# doc

[7] P.R. Lin, Y.Z. Chen, P.H. Chang and S.S. Jeng (2018). Cooperative spectrum sensing and optimization on multi-antenna energy detection in Rayleigh fading channel. 2018 27th Wireless and Optical Communication Conference (WOCC), pp. 1-5. https://doi.org/10.1109/WOCC.2018.8372735

[8] P. Anushiya and M. Suganthi (2018). Energy detection based spectrum sensing data mining for safety-message delivery in CR enabled VANET. 2018 2nd International Conference on Inventive Systems and Control (ICISC), pp. 1130-1133. https://doi.org/10.1109/ICISC.2018.8398979

[9] Giuseppe Bianchi (2000). Performance Analysis of the IEEE 802.11 Distributed Coordination Function. Communications, IEEE, vol.18, pp. 535-547,

[10] Hongning Li, Qingqi Pei, Lichuan Ma (2014). Channel Selection Information Hiding Scheme for Tracking User Attack in Cognitive Radio Networks. China Communications, pp. 125-136. 
[11] Sangeeta Singh and Aditya Trivedi(2012) Anti-jamming in Cognitive Radio Networks Using Reinforcement Learning Algorithms. Wireless and Optical Communications Networks (WOCN), 2012 Ninth International Conference on, pp. 1-5. https://doi.org/10. 1109/WOCN.2012.6331885

[12] Pradeep Kyasanur, N. H. (2006). Routing and link-layer Protocols for Multi-Channel Multi-Interface Ad Hoc Wireless Networks. SIGMOBILE Mobile Computing and Communications Review, vol. 10, no. 1, pp. 31-43. https://doi.org/10.1145/1119759.1119762

\section{$7 \quad$ Authors}

Hui Sun is with the Department of Electronic Engineering - Civil Aviation University of China, Tianjin Shi, China.

Han Han is with the College of Information Engineering and Automation, Civil Aviation University of China, Tianjin, China, 300300 (hh15821044658@163.com).

Article submitted 09 September 2018. Resubmitted 13 November 2018. Final acceptance 12 December 2018. Final version published as submitted by the author. 